\title{
Are COVID-19 vaccines safe in pregnancy?
}

Victoria Male (D)

As the COVID-19 vaccination programme starts to be rolled out, many young women are hesitant to accept the vaccine, citing concerns about fertility. Meanwhile, those offered the vaccine during pregnancy must decide whether they will accept, even though pregnant people were excluded from the clinical trials. Data on accidental pregnancies that occurred during the trials and, increasingly, outcomes in pregnant people who receive the vaccine can help these groups to make informed decisions.

In December 2020, a blog post appeared online claiming, falsely, that a senior employee at Pfizer was concerned that antibodies elicited by COVID-19 vaccines could attack the placenta. The post was quickly removed but the rumours that it started continue to spread and a survey carried out by 'Find Out Now' found that more than a quarter of young women in the United Kingdom would decline the vaccine, citing concerns about its effect on fertility. This is not the first time that unfounded rumours about vaccines causing infertility have circulated. In 2003, such concerns resulted in a boycott of polio vaccination in northern Nigeria; more recently, they have contributed to hesitance in accepting the human papillomavirus vaccine. It is understandable that people are apprehensive, especially about a new vaccine: the vast majority of adverse events can be ruled out in clinical trials, but the short time frame during which these take place, especially for COVID-19, means that events that could potentially occur decades into the future are harder to discount. Indeed, many people are hesitant specifically about receiving an mRNA vaccine, as this is a relatively new platform. With respect to these concerns, it is worth noting that the first human trials of mRNA vaccines began in 2006, so there have been 15 years during which any long-term problems arising from the platform itself could have come to light ${ }^{1}$.

Although many of the rumours that COVID-19 vaccines might damage fertility centre specifically on the mRNA platform, probably because they first emerged in the context of the Pfizer/BioNTech vaccine, the specific claim is that antibodies recognizing the SARS-CoV-2 spike protein can cross-react with the human placental protein syncytin 1 and thereby damage the placenta. If such cross-reactivity did occur, vaccines on all platforms, as well as natural infections, would be expected to be associated with placental pathology. A natural experiment assures us that this is unlikely to be the case as people who are infected with SARS-CoV-2 shortly before conceiving or early in pregnancy are no more likely to miscarry than their uninfected peers ${ }^{2}$. Nonetheless, immunologists have also taken formal approaches to address the claim that antibodies to spike protein could cross-react with syncytin 1: there is no significant similarity between the amino acid sequences of SARS-CoV-2 spike protein and syncytin 1 and convalescent serum from patients with COVID-19 does not react with syncytin 1 (REF. ${ }^{3}$.

But the data that speak most clearly to the question of whether the COVID-19 vaccines harm fertility come from the clinical trials themselves ${ }^{4-6}$. Developmental and reproductive toxicity studies show that the vaccines do not prevent female rodents becoming pregnant or harm the pups if given during pregnancy. We also have an idea of how the vaccines affect pregnancy in humans from the volunteers who became pregnant during the clinical trials. Pregnant people were excluded from the trials and participants were asked to avoid becoming pregnant, but, nonetheless, 57 pregnancies occurred across the trials of the three vaccines that have so far been approved in the UK. The outcomes of these pregnancies, so far as they have progressed, are summarized in TABLE 1 . There was no significant difference in the rate of accidental pregnancies in the vaccinated groups compared with the control groups, which indicates that the vaccines do not prevent pregnancy in humans. Similarly, the miscarriage rates are comparable between the groups, indicating no detrimental effect of vaccination on early pregnancy.

Although the data are sparse, they are so far reassuring. For this reason, regulatory bodies in the United Kingdom, European Union and United States have recommended that pregnant people should be offered the vaccine where the benefits outweigh the potential risks: pregnant workers on the frontline and those with pre-existing conditions are now receiving the vaccine. In the United States, by 10 February 2021, 20,000 pregnant people had received a COVID-19 vaccine and 
Table 1 | Accidental pregnancies in trials for the COVID-19 vaccines approved in the United Kingdom

\begin{tabular}{|c|c|c|c|c|c|c|c|}
\hline \multirow[t]{2}{*}{ Vaccine type } & \multicolumn{3}{|c|}{ Control group } & \multicolumn{3}{|c|}{ Vaccinated group } & \multirow[t]{2}{*}{ Ref. } \\
\hline & Participants & Pregnancies & $\begin{array}{l}\text { Miscarriages } \\
\text { (rate) }\end{array}$ & Participants & Pregnancies & $\begin{array}{l}\text { Miscarriages } \\
\text { (rate) }\end{array}$ & \\
\hline Pfizer/BioNTech & 18,846 & 12 & $1(8 \%)$ & 18,860 & 11 & $0(0 \%)$ & 4 \\
\hline Moderna & 15,170 & 7 & $1(14 \%)$ & 15,181 & 6 & $0(0 \%)$ & 5 \\
\hline AstraZeneca & 5,829 & 9 & $3(33 \%)$ & 5,807 & 12 & $2(17 \%)$ & 6 \\
\hline
\end{tabular}

enhanced pharmacovigilance of these vaccine recipients had raised "no red flags". In the United Kingdom, fewer people have been vaccinated, but the picture is similar.

In addition to widespread monitoring of vaccine recipients, formal studies following the outcomes for cohorts of pregnant people who receive the vaccine are underway. These are designed primarily to ensure safety and efficacy but will also address the possibility that vaccination against COVID-19 is particularly beneficial in pregnancy. Pregnant patients with COVID-19 are more likely to need intensive care, it is more likely that doctors will elect to deliver their babies early and their babies are also more likely to be admitted to the neonatal unit ${ }^{9}$. It is plausible that vaccination will reduce these risks and, should this be the case, we should consider prioritizing pregnant people for vaccination.

Further work will also address questions around whether vaccination during pregnancy has any effect on the baby. These studies will, of course, aim to rule out any detrimental effects, but many of the expected effects are beneficial. One case study found anti-spike IgG in a newborn whose mother had received the vaccine during pregnancy ${ }^{10}$ : does this occur widely and, if so, does antibody transferred across the placenta endow the infant with any protection against SARS-CoV-2 infection or COVID-19? Similarly, work is underway to determine the extent to which vaccine-elicited antibodies to spike protein enter breast milk and whether this has any protective effect for breast-fed infants.

So, is COVID-19 vaccination safe during pregnancy? The data so far suggest that it is and, given the increased risks associated with COVID-19 in pregnancy, many pregnant people have decided to accept the vaccine. By monitoring the outcomes for these people and their babies, we will soon be able to make evidence-based recommendations on whether the vaccines should be rolled out to pregnant people more widely. In the meantime, those who are planning pregnancies can rest assured that multiple strands of evidence show that vaccination does not harm fertility.

1. Pardi, N., Hogan, M. J., Porter, F. W. \& Weissman, D. mRNA vaccines - a new era in vaccinology. Nat. Rev. Drug Discov. 17 , 261-279 (2018).

2. Cosma, S. et al. Coronavirus disease 2019 and first-trimester spontaneous abortion: a case-control study of 225 pregnant patients. Am. J. Obstet. Gynecol. https://doi.org/10.1016/ j.ajog.2020.10.005 (2020)

3. Lu-Culligan, A. \& Iwasaki, A. The false rumours about vaccines that are scaring women. New York Times (26 Jan 2021).

4. United States Food \& Drug Administration. Pfizer-BioNTech COVID-19 vaccine (BNT162, PF-07302048). Vaccines and related biological products advisory committee briefing document. FDA https://www.fda.gov/media/144246/download (2020).

5. United States Food $\&$ Drug Administration. Vaccines and related biological products advisory committee meeting December 17, 2020. FDA briefing document: Moderna COVID-19 vaccine. FDA https:// www.fda.gov/media/144434/download (2020).

6. Medicines $\&$ Healthcare products Regulatory Agency. Public assessment report authorisation for temporary supply. COVID-19 vaccine AstraZeneca, solution for injection in multidose container COVID-19 vaccine (ChAdOx1-S [recombinant]). MHRA https:// assets.publishing.service.gov.uk/government/uploads/system/ uploads/attachment_data/file/949772/UKPAR_COVID_19_Vaccine_ AstraZeneca_05.01.2021.pdf (2021).

7. Nunez-Smith, M. \& Fauci, A. Press briefing by White House COVID-19 response team and public health officials, 10 February 2021. The White House https://www.whitehouse.gov/briefing-room/pressbriefings/2021/02/10/press-briefing-by-white-house-covid-19response-team-and-public-health-officials-3/ (2021).

8. Medicines $\&$ Healthcare products Regulatory Agency. Coronavirus vaccine - weekly summary of yellow card reporting. gov.uk https:// www.gov.uk/government/publications/coronavirus-covid-19-vaccineadverse-reactions/coronavirus-vaccine-summary-of-yellow-cardreporting (2021)

9. Allotey, J. et al. Clinical manifestations, risk factors, and maternal and perinatal outcomes of coronavirus disease 2019 in pregnancy: living systematic review and meta-analysis. BMJ 370, m3320 (2020).

10. Gilbert, P. \& Rudnick, C. Newborn antibodies to SARS-CoV-2 detected in cord blood after maternal vaccination. Preprint at medRxiv https://doi.org/10.1101/2021.02.03.21250579 (2021).

Competing interests

The author declares no competing interests. 